\title{
Direct observation of local chemistry and local cation displacements in the relaxor ferroelectric PMN-PT
}

\author{
Matthew J. Cabral ${ }^{1}$, Shujun Zhang ${ }^{2}$, Elizabeth C. Dickey ${ }^{1}$, and James M. LeBeau \\ 1. Department of Materials Science \& Engineering, North Carolina State University, Raleigh NC 27695 \\ 2. Institute for Superconducting and Electronic Materials, Australian Institute of Innovative Materials, \\ University of Wollongong, NSW, 2500, Australia
}

Picometer-scale lattice distortions play an important role on the macroscopic properties of relaxor ferroelectric materials. Due to competition between composition/charge ordering in these materials, there is a formation of "polar nanoregions" where local fluctuations in the B-site give rise to inhomogeneities in local strain and polarization on the nanometer scale. Direct real-space visualization of these distortions and local chemical composition can provide important insight into the formation of these "polar nanoregions".

In this talk, we present a study of local polarization and its relation with local chemistry in the relaxor ferroelectric (1-x) $\mathrm{Pb}\left(\mathrm{Mg}_{1 / 3} \mathrm{Nb}_{2 / 3}\right) \mathrm{O}_{3}-\mathrm{xPbTiO}_{3}(\mathrm{PMN}-\mathrm{PT})$. In this perovskite structured material, the $\mathrm{A}$ sub-lattice contains $\mathrm{Pb}$, while the $\mathrm{B}$ sub-lattice contains $\mathrm{Mg} / \mathrm{Nb} / \mathrm{Ti}$. Due to the complex local charge and chemical variation on the B sub-lattice, local unit cell distortions are expected. The development of the revolving scanning transmission electron microscopy (RevSTEM) technique removes sample drift from STEM images, which allows for investigation of polarization on a unit cell basis with picometer precision [1]. We show that the use of atom column indexing allows for direct measurement of local displacements within the A and B sub-lattice positions and their visualization [2]. Figure 1a illustrates the direction of local polarization of a PMN-32PT sample; with the polarization direction represented using a color wheel. The figure indicates that B-site polarization occurs along $<110>$ type directions. This agrees with the polarization direction measured using x-ray diffraction [3]. Additionally, the magnitude of the polarization was directly measured. Figure $1 \mathrm{~b}$ illustrates that the magnitude of the polarization ranges from $30 \mathrm{pm}$ at the bottom of the image to $0 \mathrm{pm}$ at the top of the image.

We also discuss the results in the context of multislice STEM image simulations to further understand the effect of polarization and overlapping domains in PMN-PT. Supercells are created with identical symmetry and B-site chemical composition as the experimental material. For the simulations, $\mathrm{Mg} / \mathrm{Nb} / \mathrm{Ti}$ are distributed on the $\mathrm{B}$ sub-lattice at their expected compositions using a binomial random distribution. Figure 2 illustrates the effect of overlapping domains of varying thickness with the polarization pointing in opposite directions. These simulations have been performed on PMN-27PT samples where polarization is expected to occur along $<111>$. It is shown that despite misoriented domains, the polarization still points in the same direction as that of the top part of the supercell. Simulations have been performed for 4, 6, 8, and $10 \mathrm{~nm}$ thick supercells with opposite polarization directions. The multislice simulations indicate that despite the change in thickness, the domain orientations resulting are strongly influenced by the atoms near the entrance surface. The $4 \mathrm{~nm}$ simulation indicates that there can be some zero net polarizations, but the overall directions remain the same. The small polarizations observed in Figure 2a will be discussed, particularly in the context of electron-channeling effects along with the influence of atom column chemistry. Finally, we will discuss how these techniques can be applied across a range of relaxors in order to understand their properties. 
References:

[1] X. Sang and J.M. LeBeau, Ultramicroscopy 138 (2014).

[2] X. Sang, A.A. Oni and J.M. LeBeau, Microscopy and Microanalysis 20 (2014).

[3] Singh, A.K. and Pandey, D. Physical Review B 67 (2003).

[4] E.J. Kirkland, et al. Ultramicroscopy 23 (1987).

[5] This material is based upon work supported by the National Science Foundation, as part of the Center for Dielectrics and Piezoelectrics under Grant Nos. IIP-1361571 and IIP-1361503. The authors acknowledge the use of the Analytical Instrumentation Facility at NCSU, which is supported by the state of North Carolina and the National Science Foundation.

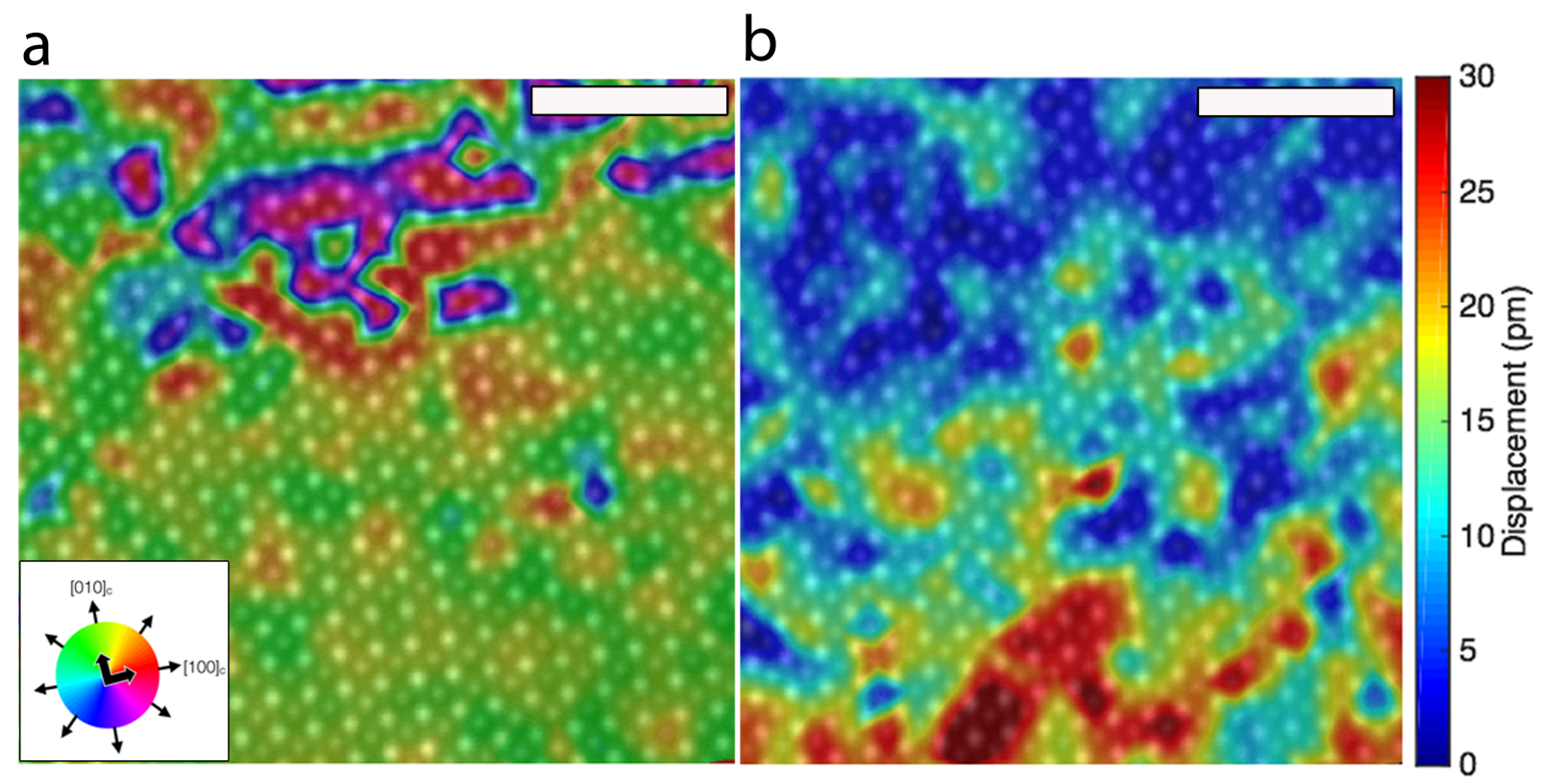

Figure 1. RevSTEM image along $<100>$ of PMN-32PT overlaid with a color map representing (a) polarization direction, see inset for colorscale, and (b) magnitude of polarization for B-site atoms. Scale bar represents $2 \mathrm{~nm}$.

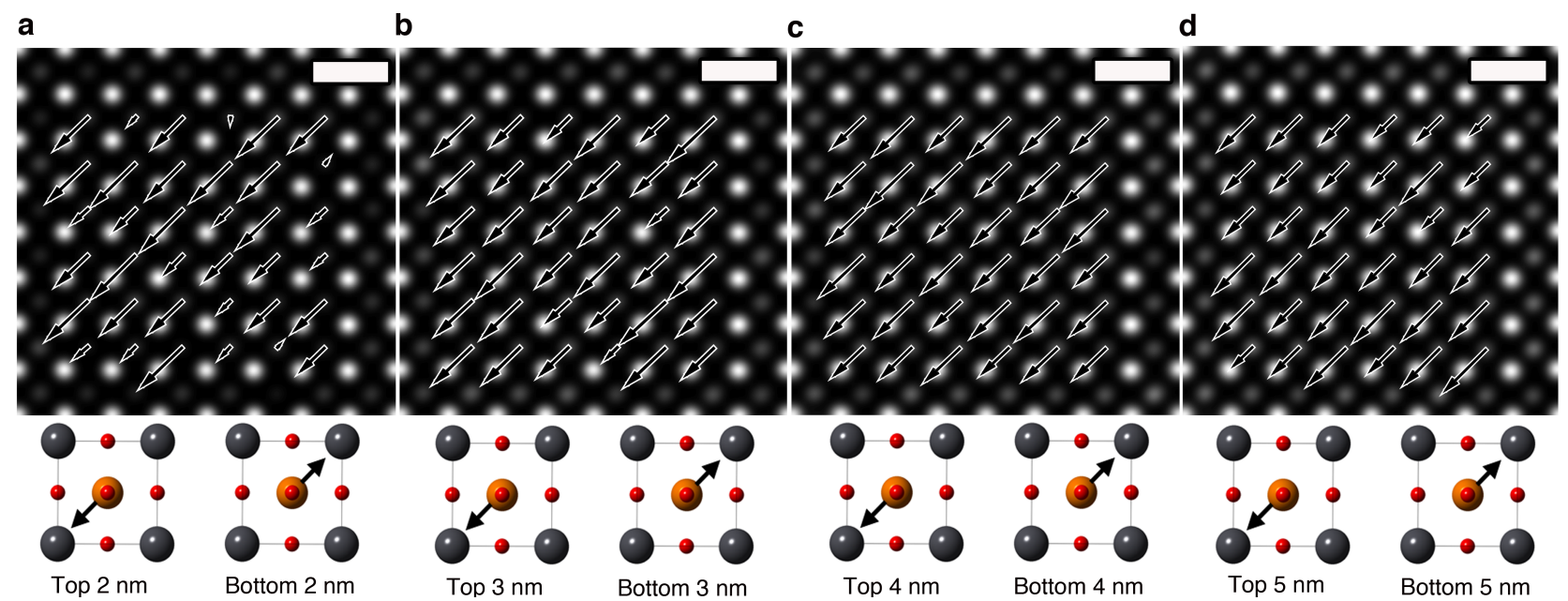

Figure 2: Multislice simulation comprising overlapping, opposite domain orientations. Total sample thicknesses are as follows: (a) 4, (b) 6, (c) 8, (d) $10 \mathrm{~nm}$. Scale bar represents $0.5 \mathrm{~nm}$. 